\title{
Attosecond x-ray transient absorption spectroscopy in graphene
}

\author{
Giovanni Cistaro, ${ }^{1}$ Luis Plaja $\odot,{ }^{2}$ Fernando Martín $\odot,{ }^{1,3,4}$ and Antonio Picón $\circledast^{1, *}$ \\ ${ }^{1}$ Departamento de Química, Universidad Autónoma de Madrid, 28049 Madrid, Spain \\ ${ }^{2}$ Grupo de Investigación en Aplicaciones del Láser y Fotónica, Departamento de Física Aplicada, \\ University of Salamanca, E-37008, Salamanca, Spain \\ ${ }^{3}$ Instituto Madrileño de Estudios Avanzados en Nanociencia (IMDEA-Nanociencia), Cantoblanco, 28049 Madrid, Spain \\ ${ }^{4}$ Condensed Matter Physics Center (IFIMAC), Universidad Autónoma de Madrid, 28049 Madrid, Spain
}

(Received 30 October 2020; accepted 27 January 2021; published 12 February 2021)

\begin{abstract}
Real-time observations of the coherent electron dynamics induced by ultrashort light pulses in condensedmatter systems have remained elusive due to limitations in existing attosecond devices. Here we show that attosecond transient absorption resulting from an IR-pump/x-ray probe numerical experiment performed in graphene unambiguously encodes the information of the coherent electron dynamics induced by the pump pulse. First, we demonstrate the possibility of tracking in real time the electron injection through the Dirac points. Second, we derive and demonstrate a simple semiclassical theory that correlates the energy structure and the action Berry phase with strong changes in the absorption spectrum around Van Hove singularities. Our work opens the way to the development of novel imaging techniques in condensed matter systems at the attosecond timescale.
\end{abstract}

DOI: 10.1103/PhysRevResearch.3.013144

\section{INTRODUCTION}

Very recently, petahertz laser fields have enabled the possibility to drive fast currents in dielectric, semiconductor, and modern materials, opening the door to a new timescale for control in solid-state systems [1-5]. In particular, the manipulation of electron dynamics at the attosecond timescale $\left(10^{-18} \mathrm{~s}\right)$, i.e., well before the lattice starts to respond to the external field, allows one to exploit electron coherence to develop applications beyond classical electronics [6-9]. In this context, real-time imaging of coherent electron dynamics is the first necessary step. Significant progress in this direction has been achieved in the last few years using high-order harmonic generation (HHG) and attosecond pumpprobe schemes.

HHG has been shown to be sensitive both to Berry phase [10-13] and valley pseudospin [14] effects, and useful to perform tomography of energy bands $[15,16]$. In HHG, the same laser field is used to induce and probe the electron dynamics occurring over approximately a laser cycle, but dynamics occurring at longer term are better investigated by using pump-probe setups. Among the latter, streaking and RABBIT (reconstruction of attosecond beatings by interference of two-photon transitions) have been used to

\footnotetext{
*Corresponding author: antonio.picon@uam.es
}

Published by the American Physical Society under the terms of the Creative Commons Attribution 4.0 International license. Further distribution of this work must maintain attribution to the author(s) and the published article's title, journal citation, and DOI. investigate the transport of electrons [17-19] and photoionization time delays [20,21]. However, due to the special characteristics of the pulses used in these scenarios (intense IR fields in the former case, trains of attosecond pulses in the latter), as well as the fact that only photoelectrons are detected, unambiguous observations of the coherent motion of electrons within these materials in real time remain to be achieved.

An alternative pump-probe scenario for solid materials (also for gas-phase studies) currently under development in several laboratories consists of using an ultrashort IR pump pulse to excite the system and a single attosecond pulse to probe the pump-induced dynamics with a controlled time delay, see Fig. 1(a). In this scheme the IR-induced dynamics produces changes in the absorption of the attosecond pulse as a function of the pump-probe delay (attosecond transient absorption spectroscopy, ATAS). The power of ATAS is that it combines attosecond temporal resolution with high energy resolution, much higher than that provided by photoelectron spectroscopy. Thus, in recent years there have been significant efforts to apply ATAS to condensed-matter systems, with remarkable achievements as the tracking in real time of carrier injection and electron-hole dynamics driven by petahertz laser fields in semiconductor, semimetal, and dielectric materials [3,22-28]. However, there still remains the problem of extracting detailed information on the quantum properties of the light-induced electron wave packet, likely because most of these works made use of XUV probe pulses, which generate in turn electron dynamics from the (usually) very dispersive inner-valence bands of the system, thus blurring the original dynamics. To avoid the additional features introduced by XUV pulses in ATAS, current experimental efforts aim at 

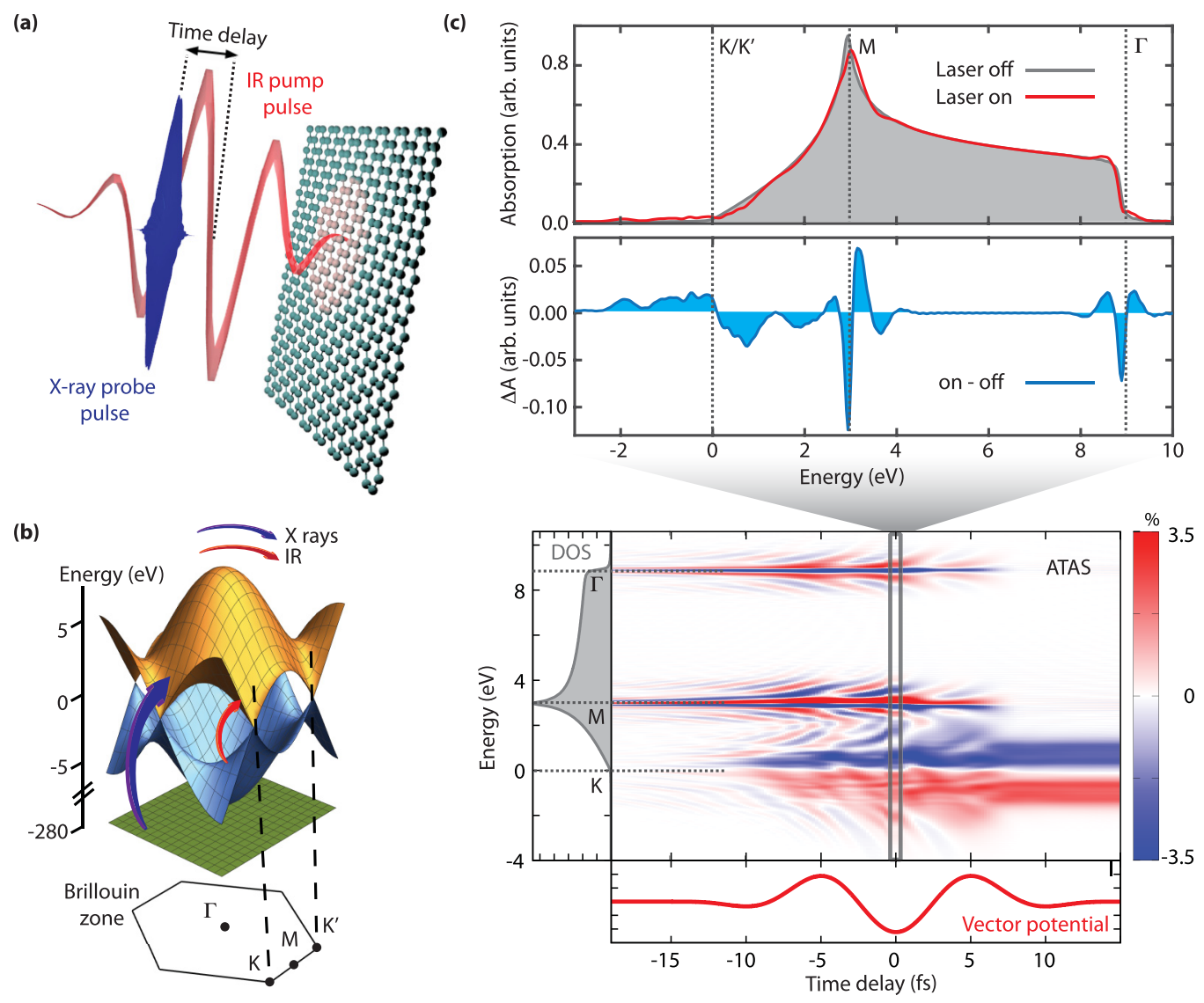

FIG. 1. Attosecond transient absorption spectroscopy. (a) Scheme of the pump-probe scheme. (b) Energy bands considered in our model. (c) X-ray attosecond transient absorption spectrum at the $K$ edge. For time delay $0 \mathrm{fs}$, the $\mathrm{x}$-ray attosecond absorption spectrum is shown with and without the IR laser pulse. The IR polarization is along the $\Gamma-M$ direction. For negative time delays the x-ray pulse arrives before the IR laser pulse. The 0 energy is referred to the Fermi level.

using high-energy XUV and x-ray probe pulses [26,28], which preferentially remove electrons from localized atomic cores, hence flat bands (i.e., no additional features that may mask the pump-induced dynamics), providing in addition element selectivity [29]. Furthermore, state-of-the-art technology will enable the production of attosecond $\mathrm{x}$-ray pulses not only at laser-based sources [30-32], but also at accelerator-based sources [33,34].

In this work we develop a semiclassical theory that allows us to correlate x-ray ATAS features with the electron dynamics. We show that ATAS changes around Van Hove points, i.e., points in the Brillouin zone (BZ) in which the density of states presents singularities, are directly associated with a coherent phase that electrons acquire during the dynamics driven by a petahertz laser field, and this phase contains the information of the semiclassical action. We derive the semiclassical action for a general system that could be described by a two-band model at the Fermi level. Interestingly, the semiclassical action is connected both to the local energy dispersion and the Berry connections of the conduction band. This is just possible due to the special characteristics of attosecond x-ray pulses, which couple flat bands (core electrons) with the conduction and valence bands. By using graphene as a benchmark, we show that ATAS resulting from an attosecond IR-pump/xray probe numerical experiment is in perfect agreement with our semiclassical theory. The present work establishes a theoretical framework for ATAS and suggests that attosecond electron dynamics imprinted in ATAS could be used to develop unprecedented imaging techniques for modern materials.

\section{THEORY FOR ELECTRON TIME DYNAMICS}

Time-dependent calculations in the x-ray regime, i.e., including core-hole states, are numerically costly even at the level of density functional theory [35]. In order to reduce the computational cost, we developed a specific method based on the density matrix propagation, the core-stateresolved Bloch model (cBE) [36], that efficiently simulates the electron dynamics. In this paper we actually present numerical calculations for ATAS in graphene at the carbon $K$ edge.

The time dynamics is computed by evolving the density matrix $\rho_{i j}=\left\langle\hat{a}_{i}^{\dagger} \hat{a}_{j}\right\rangle$ :

$$
\begin{aligned}
i \dot{\rho}_{i j}(\mathbf{k}, t)= & {\left[\epsilon_{j}(\mathbf{k})-\epsilon_{i}(\mathbf{k})-i \Gamma_{i j}\right] \rho_{i j}(\mathbf{k}, t) } \\
& +\sum_{\lambda^{\prime}} \boldsymbol{\varepsilon}(t) \cdot \boldsymbol{\xi}_{j \lambda^{\prime}}(\mathbf{k}) \rho_{i \lambda^{\prime}}(\mathbf{k}, t)
\end{aligned}
$$




$$
\begin{aligned}
& -\sum_{\lambda^{\prime}} \boldsymbol{\varepsilon}(t) \cdot \boldsymbol{\xi}_{i \lambda^{\prime}}^{*}(\mathbf{k}) \rho_{\lambda^{\prime} j}(\mathbf{k}, t) \\
& +i \boldsymbol{\varepsilon}(t) \cdot \frac{\partial}{\partial \mathbf{k}} \rho_{i j}(\mathbf{k}, t),
\end{aligned}
$$

where the electric field is the sum of both the x-ray and the IR pulse. The relaxation terms $\Gamma_{i j}$ are only different than zero for the core-hole populations and coherences. In order to evolve the density matrix we perform the rotating-wave approximation (RWA) on the x-ray interactions as it is detailed in Ref. [36]. Also, we use a basis transformation that allow us to avoid the problem of the Berry connection singularity at the Dirac points, similarly as we did in Refs. [37,38].

The attosecond absorption is obtained by the expression $[36,39]$

$$
\sigma(\omega)=\frac{8 \pi \alpha \omega}{\left|\varepsilon_{x}(\omega)\right|^{2}} \operatorname{Im}\left[\boldsymbol{\mu}(\omega) \cdot \boldsymbol{\varepsilon}_{x}^{*}(\omega)\right],
$$

where $\varepsilon_{x}^{*}(\omega)$ is the Fourier transform of the time-dependent electric field of the attosecond pulse, and $\boldsymbol{\mu}(\omega)$ is Fourier transform of the dipole response of the system calculated as

$$
\boldsymbol{\mu}(t)=q \sum_{\mathbf{k}} \sum_{i, b}\left[\boldsymbol{\xi}_{i b}(\mathbf{k}) \rho_{i b}(\mathbf{k}, t)+\text { c.c. }\right]
$$

where $q$ is the charge of the electron, $\rho_{i b}(\mathbf{k}, t)$ is the coherence term between core holes and electrons in the conduction/valence band of the density matrix, and $\boldsymbol{\xi}_{i b}(\mathbf{k})$ is the Berry connection associated with the x-ray coupling. After the x-ray excitation, the evolution of the coherence term also depends on the dynamics driven by the IR field and, therefore, on the energy dispersion and intraband Berry connection of the conduction band.

\section{ATTOSECOND TRANSIENT ABSORPTION IN GRAPHENE}

We compute the X-ray ATAS for graphene at photon energies around the carbon $K$ edge. In this scheme, the IR pulse produces the main dynamics at the bands close to the Fermi level, while the X-ray pulse couples the $1 s$ orbitals with the valence and conduction orbitals. The cBE method is used to compute the electron time dynamics [36]. We use a tight-binding model to describe graphene, as in previous Refs. [37,38], but now we also include the core orbitals of the carbon atoms, see Appendix A. We have then four energy bands in this model; two core bands, which arise from the two $1 s$ orbitals of the two carbon atoms in the unit cell, and the valence and conduction band, which arise from the $\pi$ orbitals orthogonal to the monolayer, see Fig. 1(b). Note that the two core bands are degenerate and have constant energy over the $k$ space. The core-hole lifetime is $6.1 \mathrm{fs}$ (width $\Gamma_{\mathrm{ch}}=0.108 \mathrm{eV}$ ). The X-ray pulse has a full-width half-maximum (FWHM) of 80 as and an intensity of $10^{9} \mathrm{~W} / \mathrm{cm}^{2}$, which is low enough to consider its interaction with graphene in the weak regime. The X-ray photon energy is tuned at the Fermi level, around $280 \mathrm{eV}$. We consider Fourier-transform-limited pulses, and the bandwidth covers both valence and conduction band. The polarization is perpendicular to the graphene monolayer. The IR pulse has a wavelength of $3 \mu \mathrm{m}$ (photon energy $0.41 \mathrm{eV}$ ), and a FWHM of $\sim 7.2 \mathrm{fs}$, that is a three-cycle pulse with a $\operatorname{sinc}^{2}$ envelope. The IR intensity is $10^{11} \mathrm{~W} / \mathrm{cm}^{2}$, strong enough to produce significant intraband dynamics in graphene and avoid material damage [37]. The IR polarization is in the plane of the monolayer and along the direction of the $\Gamma-M$ points unless stated differently.

The X-ray ATAS around the Fermi level is shown in Fig. 1(c). Initially, the valence band is occupied and core electrons can only be promoted into the conduction band [top panel Fig. 1(c)]. When the IR is not present, the absorption spectrum is proportional to the density of states in the conduction band, this is what is expected at first-order perturbation theory for low-intensity x-ray pulses. We observe three important regions in the ATAS spectrum: (i) around the $K / K^{\prime}$ point located at $0 \mathrm{eV}$, where the static (laser-off) spectrum starts to rise, (ii) around the $M$ point, where the static spectrum presents a strong peak at $\sim 3 \mathrm{eV}$, and (iii) around the $\Gamma$ point, where the static spectrum drops at $\sim 9 \mathrm{eV}$. To analyze the IR induced dynamics in these three regions, we show the absorption spectrum for a time delay 0 fs [the IR and $\mathrm{X}$-ray pulses maximally overlap, middle panel Fig. 1(c)]. The changes around the $K / K^{\prime}$ point are produced by the promotion of electrons from the valence to the conduction band by the IR pulse. When a hole is created in the valence band, core electrons are allowed to fill the created hole, and this ends in an absorption increase for energies below $0 \mathrm{eV}$. In our calculations, the injected charge corresponds around $1 \%$ of the valence charge. Similarly, when the valence electron is promoted into the conduction band, this occupies a state in the $k$ space and blocks core electrons to be excited to such state. This then ends in an absorption decrease. Interestingly, the absorption also presents some interference patterns at negative and small positive delays [lower panel in Fig. 1(c)], most likely due to the complex dynamics of carriers traversing the Dirac points, which play a crucial role in the first step of $\mathrm{HHG}$ in graphene [37]. Now, the energies corresponding to the $M$ and $\Gamma$ points are quite far from the Fermi level, and the direct excitation between valence and conduction band requires several photons to promote a significant population. Even though these points show important changes in the absorption spectrum. These features vanish at 5 fs time delay, when the vector potential starts to decrease after the creation of the core hole, a quite different behavior from that observed at the Dirac points.

Previous experimental and theoretical studies in condensed-matter systems [24,27,36,40] have shown that IR laser fields, as the one used in the present work, induce a significant intraband dynamics, so we expect that this dynamics also reflects in the ATAS. Indeed, when core electrons are promoted to the conduction band by the $\mathrm{x}$-ray pulse, electrons are further driven by the IR field along the energy band. This coherent dynamics results in a dynamical Stark shift effect that changes the attosecond absorption line shape profile [36], similarly to the effects found in atomic systems [41,42]. The features observed around the $M$ and $\Gamma$ points exhibit recurrences as a function of time delay and have a geometrical origin as in atomic and molecular systems [39,42,43]. However, we will show that these recurrences encode richer information in condensed-matter systems. Indeed, note that while the recurrent peaks are quite symmetric with respect to the energy of the gamma point, 

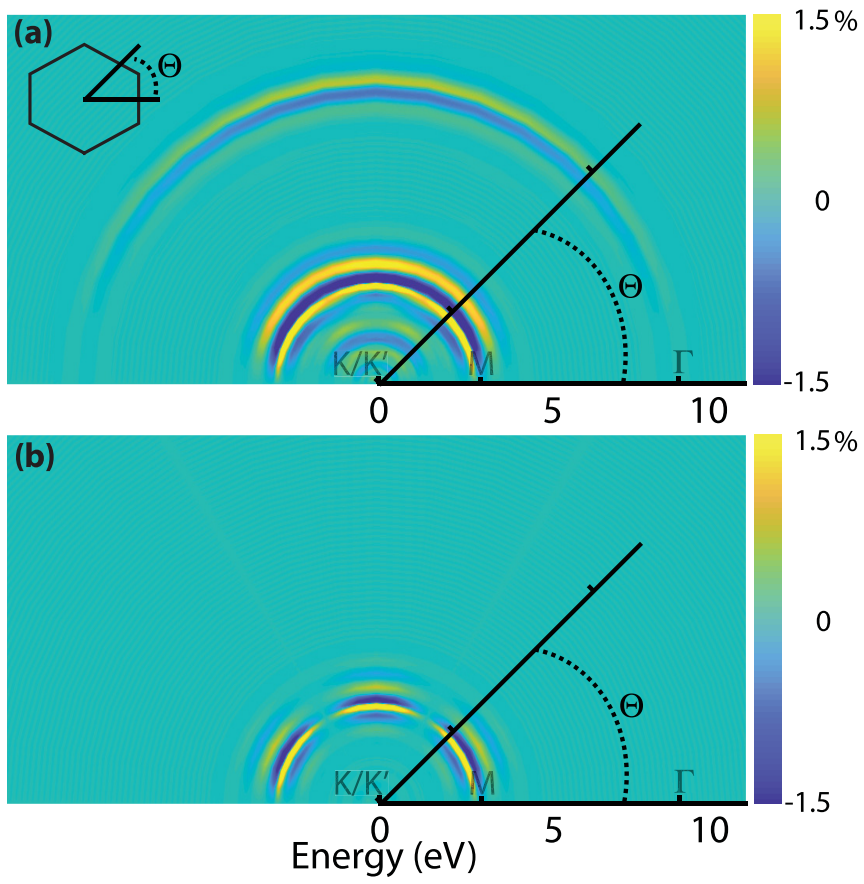

FIG. 2. IR polarization dependence at Van Hove singularities. (a) Relative difference between the absorption at a particular angle $\Theta$ and the absorption at $\Theta=0$, where the angle $\Theta$ is the direction of the IR polarization with respect to the $\Gamma-M$ direction. The two pulses are overlapping at 0 fs time delay. (b) The same as (a), but the calculations do not consider the effects arising from the Berry connections, see main text.

those peaks are antisymmetric with respect to the energy of the $M$ point.

If the intraband dynamics is the underlying mechanism of the $M$ and $\Gamma$ features, the transient absorption shown in Fig. 1(c) should be sensitive to the IR polarization direction, as the energy landscape traveled by the core electron in the conduction band depends on the direction of the electronic motion. In Fig. 2(a) we show the absorption spectrum for different IR polarization directions and for a fixed 0 fs time delay. The angle $\Theta$ corresponds to the direction of the IR polarization, where $\Theta=0$ is the direction along the $\Gamma-M$ points in the Brillouin zone. In order to visualize the changes due to the IR polarization, the difference between the absorption at a particular angle and the absorption at $\Theta=0$ is taken. We observe that the changes are well localized around the $M$ and $\Gamma$ points, i.e., in Van Hove singularities in which the energy gradient with respect to the quasimomentum is zero, see Fig. 1(b).

If the energy dispersion plays the dominant role in the features observed in Fig. 2(a), we should expect that those preserve the symmetry of the crystal, but it is not the case. We repeat the calculations of Fig. 2(a), but now considering constant Berry connections in the eigenstate basis, so the time evolution of the coherence is modified only by the $k$ dependency of the energy band, see Fig. 2(b). In this case the absorption features clearly preserve the symmetry of the crystal. Now the $\Gamma$ point does not show any changes, as around that point the energy structure is the same for any direction. At the Dirac points, as the Berry connection is constant, also the excitation between valence and conduction band does not depend on the IR polarization direction. These calculations really indicate the importance of the Berry connection effects in the electron dynamics and, consequently, in the absorption spectrum.

\section{SEMICLASSICAL MODEL FOR ATAS}

In order to gain further insight to the nature of the recurrent peaks and the angle-dependence effects, we develop a semiclassical theory that allow us to correlate the IR-induced electron dynamics to the observed ATAS features. This theory is general for any material that can be described with two energy bands around the Fermi level. Interestingly, the theory clearly reflects the contribution in ATAS of not only the energy dispersion, but also of the Berry connections. We consider a general two-band Hamiltonian written as $H(\mathbf{k})=$ $B_{0}(\mathbf{k}) 1+B_{x}(\mathbf{k}) \sigma_{x}+B_{y}(\mathbf{k}) \sigma_{y}+B_{z}(\mathbf{k}) \sigma_{z}$, where 1 is the identity matrix and $\sigma_{i}$ are the Pauli matrices. In this approach, the time-dependent dipole of the system is calculated by the sum of semiclassical electron trajectories driven by the IR vector potential. The approach assumes that an electron starts in a particular $\mathbf{k}$ point of the conduction band, i.e., the excitation by the attosecond pulse is sudden, and the quasimomentum evolves as $\mathbf{K}=\mathbf{k}-\mathbf{A}(t)$. Along this trajectory, the electron acquires a phase given by

$$
\begin{aligned}
\Delta \varphi_{ \pm}\left(t, t_{0}, \mathbf{K}\right)= & i \int_{t_{0}}^{t} d t^{\prime}\left(\left\{\epsilon_{c}\left[\mathbf{K}+\mathbf{A}\left(t^{\prime}\right)\right]-\epsilon_{c}(\mathbf{K})\right\}\right) \\
& +i \int_{t_{0}}^{t} d t^{\prime}\left\{\Phi_{S \pm}\left[\mathbf{K}+\mathbf{A}\left(t^{\prime}\right), t^{\prime}\right]\right\},
\end{aligned}
$$

where the action Berry phase $\Phi_{S \pm}$ is defined as $\Phi_{S \pm}(\mathbf{k}, t)=$ $\frac{1}{2} \boldsymbol{\varepsilon}_{I R}(t) \cdot \partial_{\mathbf{k}} \phi(\mathbf{k})[\cos \theta(\mathbf{k}) \pm 1]$, where $\epsilon_{c}(\mathbf{k})$ is the energy of the conduction band, $\tan \phi(\mathbf{k})=B_{y}(\mathbf{k}) / B_{x}(\mathbf{k})$, and $\cos \theta(\mathbf{k})=B_{z}(\mathbf{k}) / \sqrt{B_{x}^{2}(\mathbf{k})+B_{y}^{2}(\mathbf{k})+B_{z}^{2}(\mathbf{k})}$, see more details in Appendix B. The two different signs in the action phase is because the electron could be excited from two different core orbitals into the conduction band. Note that the coherent phase mainly depends on the conduction band and not at all on the core-hole band. This is due to the fact that the core orbitals are well localized and, therefore, the electronic structure is not $k$ dependent. The action Berry phase $\Phi_{S}$ arises from the interband Berry connection from the flat band to the conduction band and the intraband Berry connection in the conduction band, and it is gauge invariant [13].

Once we calculate the phase for different trajectories at different points of the $k$ space, then we calculate the timedependent dipole as

$$
\boldsymbol{\mu}(t) \propto \sum_{\mathbf{K}, \pm}\left[e^{-i\left[\epsilon_{\mathrm{ch}}(\mathbf{K})-\epsilon_{c}(\mathbf{K})-i \Gamma_{\mathrm{ch}} / 2\right]\left(t-t_{0}\right)+\Delta \varphi_{ \pm}\left(t, t_{0}, \mathbf{K}\right)}+\text { c.c. }\right] .
$$

The previous equation can be easily understood as the sum of all the oscillating dipole terms in the reciprocal space, which deviates by a phase $\Delta \varphi_{ \pm}$that arises from the light-induced electron motion during the core-hole decay. We use Eq. (5) to calculate the attosecond transient absorption spectrum and the absorption spectrum vs the polarization angle. The results 


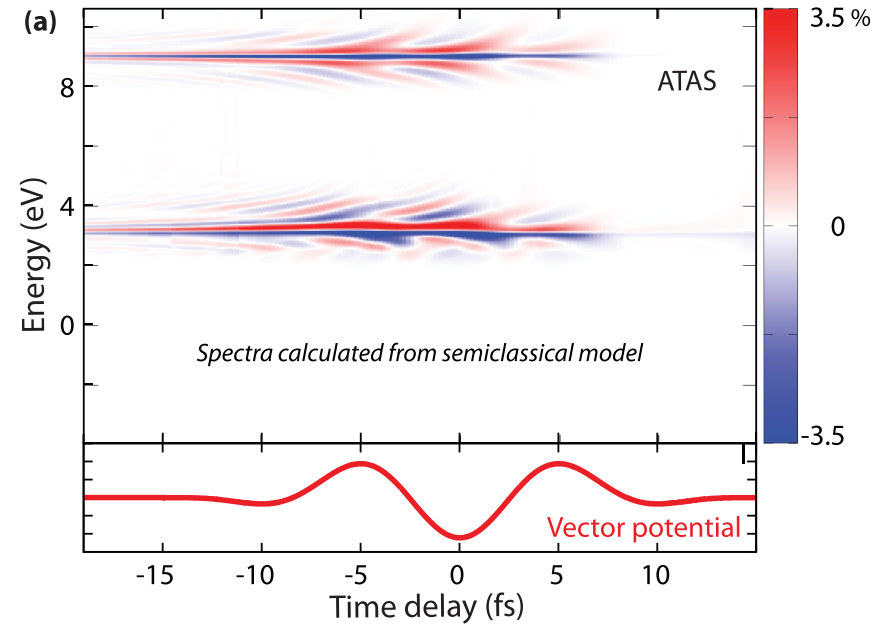

(b)

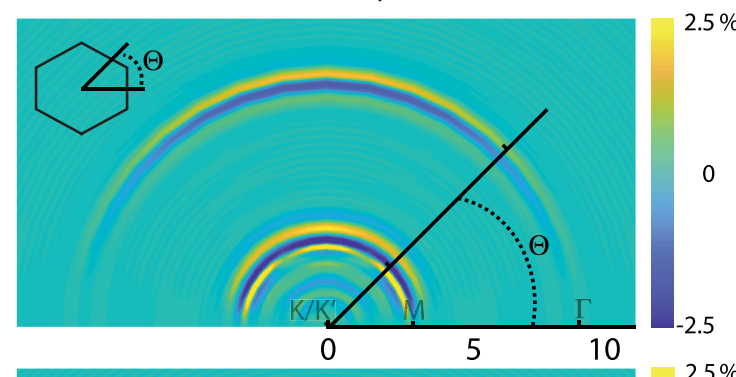

(c)

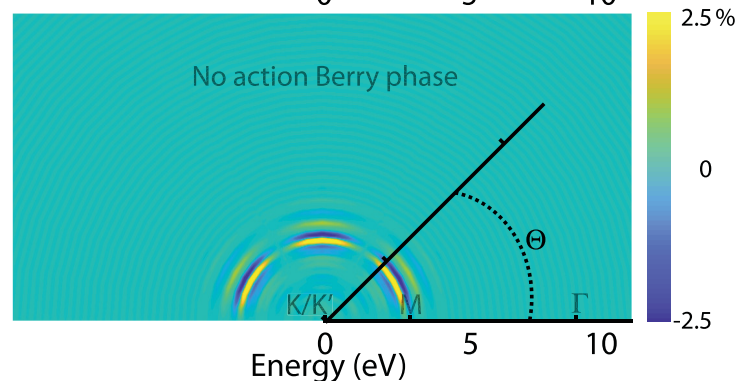

FIG. 3. Semiclassical model. (a) ATAS calculated with the semiclassical model. (b) and (c) The same as Fig. 2 but using the semiclassical model.

shown in Fig. 3 are in perfect agreement with the numerical cBE ones reported in Figs. 1 and 2. We would like to emphasize that even though the sum in Eq. (5) is over the whole BZ, the changes are localized around the Van Hove singularities. The recurrent ATAS features are dominated by the energy term of Eq. (4). The symmetric and antisymmetric pattern in the $\Gamma$ and $M$ points, respectively, results from the local energy structure: a paraboloid energy surface around the $\Gamma$ point and a hyperbolic paraboloid energy surface around the $M$ point. Contribution of the action Berry phase $\Phi_{S}$, related to the local Berry connections, to the polarization-angle absorption spectrum shown in Fig. 2(a) [or Fig. 3(b)] is of the order of $2 \%$. Interestingly, when the laser polarization is circular, the action Berry phase can be related to the Berry curvature. Investigations on the possibility to use ATAS for obtaining local information about the Berry curvature are currently in progress.

\section{CONCLUSIONS}

In summary, we have computed the ATAS spectrum of graphene for a pump-probe scheme of a few-cycle petahertz laser field and an attosecond x-ray pulse tuned at the carbon $K$ edge. We observe two interesting features in the spectrum: the injection in real time of carriers through the Dirac points and a complex pattern around the Van Hove singularities, i.e., the $M$ and $\Gamma$ points of graphene. We develop a semiclassical theory that demonstrates that the complex pattern around the Van Hove singularities is connected to a coherent phase that electrons acquire during the dynamics driven by a petahertz laser field, and this phase contains the information of the semiclassical action. We derive the semiclassical action for a general system that could be described by a two-band model at the Fermi level, and it depends both on the local energy dispersion and the local Berry connections of the conduction band. This finding opens a new route for attosecond condensed-matter science to directly access light-induced quantum properties in particular regions of the $\mathrm{BZ}$.

\section{ACKNOWLEDGMENTS}

G.C. and A.P. acknowledge funding from Comunidad de Madrid through TALENTO Grant 2017-T1/IND-5432 and computer resources and technical support from MareNostrum (RES-FI-2020-1-0005), Caesaraugusta (RES- FI-20203-0008), and CCC-UAM. A.P. acknowledges funding from Grant RTI2018-097355-A-I00 (MCIU/AEI/FEDER, UE). L.P. acknowledges support from Junta de Castilla y León (Project SA287P18), FEDER funds and MINECO (FIS201675652-P, PID2019-106910GB-I00). F.M. acknowledges the MICINN project PID2019-105458RB-I00, the Severo Ochoa Programme for Centres of Excellence in R\&D (SEV-20160686), and the María de Maeztu Programme for Units of Excellence in R\&D (MDM-2014-0377).

\section{APPENDIX A: TWO-BAND SYSTEM HAMILTONIAN AND CORE-ORBITALS BERRY CONNECTIONS}

In general, the Hamiltonian of a two-band system, in the $\mathbf{k}$ representation, can always be expressed as

$$
H_{w}(\mathbf{k})=B_{0}(\mathbf{k}) 1+B_{x}(\mathbf{k}) \sigma_{x}+B_{y}(\mathbf{k}) \sigma_{y}+B_{z}(\mathbf{k}) \sigma_{z},
$$

where $\sigma_{i}$ stands for the Pauli matrices and $B_{i}$ are real functions in the reciprocal space. The eigenvalues are given by $E_{ \pm}(\mathbf{k})=$ $B_{0}(\mathbf{k}) \pm \sqrt{B_{x}^{2}(\mathbf{k})+B_{y}^{2}(\mathbf{k})+B_{z}^{2}(\mathbf{k})}$ and the eigenvectors are

$$
\begin{gathered}
\left|u_{+, \mathbf{k}}\right\rangle=\left(\begin{array}{c}
e^{-i \phi(\mathbf{k}) / 2} \cos \frac{\theta(\mathbf{k})}{2} \\
e^{+i \phi(\mathbf{k}) / 2} \sin \frac{\theta(\mathbf{k})}{2}
\end{array}\right), \\
\left|u_{-, \mathbf{k}}\right\rangle=\left(\begin{array}{c}
e^{-i \phi(\mathbf{k}) / 2} \sin \frac{\theta(\mathbf{k})}{2} \\
-e^{+i \phi(\mathbf{k}) / 2} \cos \frac{\theta(\mathbf{k})}{2}
\end{array}\right),
\end{gathered}
$$


where we have defined

$$
\begin{aligned}
\tan \phi(\mathbf{k}) & =\frac{B_{y}(\mathbf{k})}{B_{x}(\mathbf{k})}, \\
\cos \theta(\mathbf{k}) & =\frac{B_{z}(\mathbf{k})}{\sqrt{B_{x}^{2}(\mathbf{k})+B_{y}^{2}(\mathbf{k})+B_{z}^{2}(\mathbf{k})}} .
\end{aligned}
$$

We consider now the previous Hamiltonian, a two-band system, coupled with core orbitals. As a core orbital is well localized in one atomic site of the system, we assume that the x-ray pulse would mainly couple orbitals localized around the same site. For graphene, we have to additionally consider that we have two energy-degenerate core orbitals. In this case we write the Berry connections as

$$
\boldsymbol{\xi}_{w}=\left(\begin{array}{cccc}
0 & 0 & \mathbf{r}_{a} & 0 \\
0 & 0 & 0 & \mathbf{r}_{b} \\
\mathbf{r}_{a} & 0 & 0 & 0 \\
0 & \mathbf{r}_{b} & 0 & 0
\end{array}\right)
$$

where we have defined $\mathbf{r}_{i}$ as the Berry connection at site $i$ between the core orbital and the outer orbital that forms the chemical bond. The outer orbital could be described by an atomic orbital, as in a tight-binding model, or a Wannier orbital. In tight-binding graphene, $\mathbf{r}_{i}$ represents the Berry connection between the $1 s$ orbital and the $2 p$ orbital (perpendicular to the surface) at each carbon atom. The Berry connections between outer orbitals are neglected. The Berry connections in the basis of the eigenvectors is then given by

$$
\boldsymbol{\xi}(\mathbf{k})=\left(\begin{array}{cccc}
0 & 0 & \mathbf{r}_{a} e^{-i \phi(\mathbf{k}) / 2} \sin \frac{\theta(\mathbf{k})}{2} & \mathbf{r}_{a} e^{-i \phi(\mathbf{k}) / 2} \cos \frac{\theta(\mathbf{k})}{2} \\
0 & 0 & -\mathbf{r}_{b} e^{+i \phi(\mathbf{k}) / 2} \cos \frac{\theta(\mathbf{k})}{2} & \mathbf{r}_{b} e^{+i \phi(\mathbf{k}) / 2} \sin \frac{\theta(\mathbf{k})}{2} \\
\mathbf{r}_{a} e^{+i \phi(\mathbf{k}) / 2} \sin \frac{\theta(\mathbf{k})}{2} & -\mathbf{r}_{b} e^{-i \phi(\mathbf{k}) / 2 \cos \frac{\theta(\mathbf{k})}{2}} & -\frac{1}{2} \partial_{\mathbf{k}} \phi(\mathbf{k}) \cos \theta(\mathbf{k}) & -i \frac{1}{2} \partial_{\mathbf{k}} \theta(\mathbf{k})+\frac{1}{2} \partial_{\mathbf{k}} \phi(\mathbf{k}) \sin \theta(\mathbf{k}) \\
\mathbf{r}_{a} e^{+i \phi(\mathbf{k}) / 2} \cos \frac{\theta(\mathbf{k})}{2} & \mathbf{r}_{b} e^{-i \phi(\mathbf{k}) / 2} \sin \frac{\theta(\mathbf{k})}{2} & i \frac{1}{2} \partial_{\mathbf{k}} \theta(\mathbf{k})+\frac{1}{2} \partial_{\mathbf{k}} \phi(\mathbf{k}) \sin \theta(\mathbf{k}) & \frac{1}{2} \partial_{\mathbf{k}} \phi(\mathbf{k}) \cos \theta(\mathbf{k})
\end{array}\right) .
$$

For the particular case of tight-binding graphene, we account for the $2 p_{z}$ atomic orbital, perpendicular to the monolayer, and the $1 s$ atomic orbital of the two carbons in the unit cell. Assuming that the $1 s$ orbitals are well localized and there is no overlapping among them, the eigenenergies for the core bands are given by $\epsilon_{\mathrm{ch}}=\epsilon_{1 s}-\epsilon_{2 p_{z}}$, and the Hamiltonian describing the valence and conduction bands is given by $B_{x}(\mathbf{k})=\gamma \operatorname{Re}[f(\mathbf{k})], B_{y}(\mathbf{k})=\gamma \operatorname{Im}[f(\mathbf{k})], B_{z}(\mathbf{k})=$ 0 , and $B_{0}(\mathbf{k})=0$. The form factor is $f(\mathbf{k})=1+e^{i \mathbf{k} \cdot \mathbf{a}_{1}}+$ $e^{i \mathbf{k} \cdot \mathbf{a}_{2}}$, where $\mathbf{a}_{i}$ are the two lattice vectors of graphene, $\mathbf{a}_{1}=\frac{a}{2}(\sqrt{3}, 1)$ and $\mathbf{a}_{2}=\frac{a}{2}(\sqrt{3},-1)$, with $a=2.46 \AA$ the lattice constant. The hopping parameter is $\gamma=2.97 \mathrm{eV}$. The eigenenergies are therefore $E(\mathbf{k})= \pm \gamma|f(\mathbf{k})|$. Using the eigenstates with the previously introduced notation, we find the Berry connection to be

$$
\boldsymbol{\xi}(\mathbf{k})=\frac{\mathbf{r}_{x}}{\sqrt{2}}\left[\begin{array}{cccc}
0 & 0 & e^{-i \phi(\mathbf{k}) / 2} & e^{-i \phi(\mathbf{k}) / 2} \\
0 & 0 & -e^{i \phi(\mathbf{k}) / 2} & e^{i \phi(\mathbf{k}) / 2} \\
e^{i \phi(\mathbf{k}) / 2} & -e^{-i \phi(\mathbf{k}) / 2} & 0 & 0 \\
e^{i \phi(\mathbf{k}) / 2} & e^{-i \phi(\mathbf{k}) / 2} & 0 & 0
\end{array}\right],
$$

where $\phi(\mathbf{k})$ is the argument of the form factor $f(\mathbf{k})$. Note that this matrix is equivalent to the general one when $\theta=\frac{\pi}{2}$ and $r_{a}=r_{b}=r_{x}$. The X-ray coupling depends on the electric dipole element between the $1 s$ and $2 p_{z}$ orbitals:

$$
\mathbf{r}_{x}=\int d^{3} r \varphi_{1 s}^{*}(\mathbf{r}) \mathbf{r} \varphi_{2 p_{z}}(\mathbf{r}) .
$$

In our case $\mathbf{r}_{x}$ is only nonzero in the direction perpendicular to the monolayer, and it is equal to $0.041 \AA$.

\section{APPENDIX B: DERIVATION OF THE SEMICLASSICAL MODEL FOR ATAS}

We present here the derivation of Eq. (5) of the main text, which is used to get a simple physical interpretation of the results obtained from the core-state-resolved Bloch model. We start deriving the light-induced dipole response of the system by using the following ansatz:

$$
\begin{aligned}
|\Psi(t)\rangle= & \sum_{\mathbf{K}}\left[b_{0}(\mathbf{K}, t)|g, \mathbf{K}\rangle+b_{1}(\mathbf{K}, t)|1, \mathbf{K}\rangle\right. \\
& \left.+b_{2}(\mathbf{K}, t)|2, \mathbf{K}\rangle\right],
\end{aligned}
$$

where $|g, \mathbf{K}\rangle=a_{\mathrm{ch} 1}^{\dagger}(\mathbf{K}) a_{\mathrm{ch} 2}^{\dagger}(\mathbf{K})|0\rangle, \quad|1, \mathbf{K}\rangle=a_{c}^{\dagger}(\mathbf{K}) a_{\mathrm{ch} 1}(\mathbf{K})$ $|g, \mathbf{K}\rangle$, and $|2, \mathbf{K}\rangle \stackrel{\cos }{=} a_{c}^{\dagger}(\mathbf{K}) a_{\mathrm{ch} 2}(\mathbf{K})|g, \mathbf{K}\rangle$, and where $a_{\alpha}^{\dagger}(\mathbf{k})$ is the operator that creates a particle with quasimomentum $\mathbf{k}$ in the $\alpha$ band.

We calculate the time-dependent evolution of the amplitudes by solving the time-dependent Schrödinger equation. The Hamiltonian is written as $H(t)=H_{0}+V_{I}(t)$, where $H_{0}$ is the free Hamiltonian and $V_{I}(t)$ is the coupling between the electric field and the system in the length gauge. Assuming a sudden excitation at $t=t_{0}$ of the x rays, $\boldsymbol{\varepsilon}_{x}(t)=\boldsymbol{\varepsilon}_{x 0} \delta\left(t-t_{0}\right)$, and the evolution of the amplitudes will be governed by the 
adiabatic solutions:

$$
\begin{aligned}
& b_{0}(\mathbf{K}, t)=e^{-i \int_{t_{0}}^{t} d t^{\prime}\left\{E_{0}\left[\mathbf{K}+\mathbf{A}\left(t^{\prime}\right)\right]+\boldsymbol{\varepsilon}\left(t^{\prime}\right) \cdot \tilde{\boldsymbol{\xi}}_{00}\left[\mathbf{K}+\mathbf{A}\left(t^{\prime}\right)\right]\right\}} b_{0}\left(\mathbf{K}, t_{0}\right), \\
& b_{1}(\mathbf{K}, t)=e^{-i \int_{t_{0}}^{t} d t^{\prime}\left\{E_{1}\left[\mathbf{K}+\mathbf{A}\left(t^{\prime}\right)\right]-i \Gamma_{\mathrm{ch}} / 2+\boldsymbol{\varepsilon}\left(t^{\prime}\right) \cdot \tilde{\xi}_{11}\left[\mathbf{K}+\mathbf{A}\left(t^{\prime}\right)\right]\right\}} b_{1}\left(\mathbf{K}, t_{0}\right), \\
& b_{2}(\mathbf{K}, t)=e^{-i \int_{t_{0}}^{t} d t^{\prime}\left\{E_{2}\left[\mathbf{K}+\mathbf{A}\left(t^{\prime}\right)\right]-i \Gamma_{\mathrm{ch}} / 2+\boldsymbol{\varepsilon}\left(t^{\prime}\right) \cdot \tilde{\xi}_{22}\left[\mathbf{K}+\mathbf{A}\left(t^{\prime}\right)\right]\right\}} b_{2}\left(\mathbf{K}, t_{0}\right),
\end{aligned}
$$

where $\Gamma_{\mathrm{ch}}$ refers to the width of the core-excited states, $\tilde{\xi}_{i i}$ are the diagonal terms of the Berry connections, $E_{i}$ are the corresponding energies of the states, and $\boldsymbol{\varepsilon}(t)$ is the external laser field. Using the correlation between time-dependent amplitudes and the coherence terms in the semiconductor Bloch equations [36], the dipole response is written as

$$
\mu(t) \propto q \sum_{\mathbf{K}} \sum_{i=1}^{2} \tilde{\boldsymbol{\xi}}_{i 0}[\mathbf{K}+\mathbf{A}(t)] b_{i}^{*}(\mathbf{K}, t) b_{0}(\mathbf{K}, t)+\text { c.c. },
$$

where $\tilde{\xi}_{i 0}$ is the off-diagonal terms of the Berry connections representing the coupling of the core-excited states with the ground state. We can rewrite the last expression as

$$
\mu(t) \propto \sum_{\mathbf{K}, i=1,2} \frac{\left|\tilde{\boldsymbol{\xi}}_{i 0}[\mathbf{K}+\mathbf{A}(t)]\right|}{\left|\tilde{\boldsymbol{\xi}}_{i 0}\left[\mathbf{K}+\mathbf{A}\left(t_{0}\right)\right]\right|} e^{-i S_{i 0}\left(\mathbf{K}, t, t_{0}\right)} e^{-\frac{\Gamma_{\mathrm{ch}}\left(t-t_{0}\right)}{2}} \tilde{\rho}_{i 0}\left(\mathbf{K}, t_{0}\right)+\text { c.c. }
$$

where we have defined

$$
\begin{aligned}
& \tilde{\rho}_{i 0}\left(\mathbf{K}, t_{0}\right)=\tilde{\boldsymbol{\xi}}_{i 0}\left[\mathbf{K}+\mathbf{A}\left(t_{0}\right)\right] b_{i}^{*}\left(\mathbf{K}, t_{0}\right) b_{0}\left(\mathbf{K}, t_{0}\right), \\
& \tilde{\boldsymbol{\xi}}_{i 0}(\mathbf{k})=\left|\tilde{\boldsymbol{\xi}}_{i 0}(\mathbf{k})\right| e^{i \varphi_{i 0}(\mathbf{k})}, \\
& S_{i 0}\left(\mathbf{K}, t, t_{0}\right)=\int_{t_{0}}^{t} d t^{\prime}\left[E_{0}\left(\mathbf{K}^{\prime}\right)-E_{i}\left(\mathbf{K}^{\prime}\right)+\boldsymbol{\varepsilon}\left(t^{\prime}\right) \cdot \tilde{\boldsymbol{\xi}}_{00}\left(\mathbf{K}^{\prime}\right)-\boldsymbol{\varepsilon}\left(t^{\prime}\right) \cdot \tilde{\boldsymbol{\xi}}_{i i}\left(\mathbf{K}^{\prime}\right)-\frac{d}{d t^{\prime}} \varphi_{i 0}\left(\mathbf{K}^{\prime}\right)\right],
\end{aligned}
$$

and $\mathbf{K}^{\prime}=\mathbf{K}+\mathbf{A}\left(t^{\prime}\right)$. The last term $S_{i 0}\left(\mathbf{K}, t, t_{0}\right)$ is the semiclassical action and it can be proven to be gauge invariant, as it is derived in Ref. [13]. Using the previous two-band Hamiltonian and the corresponding Berry connections for core orbitals, we associate $\tilde{\boldsymbol{\xi}}_{01}=\boldsymbol{\xi}_{1,4}, \tilde{\boldsymbol{\xi}}_{02}=\boldsymbol{\xi}_{2,4}, \tilde{\boldsymbol{\xi}}_{00}=\boldsymbol{\xi}_{1,1}+\boldsymbol{\xi}_{2,2}, \tilde{\boldsymbol{\xi}}_{11}=\boldsymbol{\xi}_{4,4}-\boldsymbol{\xi}_{1,1}$, and $\tilde{\boldsymbol{\xi}}_{22}=\boldsymbol{\xi}_{4,4}-\boldsymbol{\xi}_{2,2}$.

The energies are defined by $E_{0}(\mathbf{k})=\epsilon_{\mathrm{ch}_{1}}(\mathbf{k})+\epsilon_{\mathrm{ch}_{2}}(\mathbf{k}), E_{1}(\mathbf{k})=\epsilon_{c}(\mathbf{k})+\epsilon_{\mathrm{ch}_{2}}(\mathbf{k})$, and $E_{2}(\mathbf{k})=\epsilon_{c}(\mathbf{k})+\epsilon_{\mathrm{ch}_{1}}(\mathbf{k})$, where $\epsilon_{c}, \epsilon_{\mathrm{ch}_{1}}$, and $\epsilon_{\mathrm{ch}_{2}}$ are the orbital energies for the conduction state and core-excited states, respectively. The energies of the core-excited states are degenerate $\epsilon_{\mathrm{ch}_{1}}=\epsilon_{\mathrm{ch}_{2}}$ and do not depend on $\mathbf{k}$, and $\epsilon_{c}(\mathbf{k})=E_{+}(\mathbf{k})$. For the two-band system the action functions take the form

$$
\begin{aligned}
& S_{10}\left(\mathbf{K}, t, t_{0}\right)=\int_{t_{0}}^{t} d t^{\prime}\left[\epsilon_{\mathrm{ch}_{1}}\left(\mathbf{K}^{\prime}\right)-\epsilon_{c}\left(\mathbf{K}^{\prime}\right)-\frac{1}{2} \boldsymbol{\varepsilon}\left(t^{\prime}\right) \cdot \partial_{\mathbf{k}} \phi\left(\mathbf{K}^{\prime}\right)\left[\cos \theta\left(\mathbf{K}^{\prime}\right)-1\right]\right], \\
& S_{20}\left(\mathbf{K}, t, t_{0}\right)=\int_{t_{0}}^{t} d t^{\prime}\left[\epsilon_{\mathrm{ch}_{2}}\left(\mathbf{K}^{\prime}\right)-\epsilon_{c}\left(\mathbf{K}^{\prime}\right)-\frac{1}{2} \boldsymbol{\varepsilon}\left(t^{\prime}\right) \cdot \partial_{\mathbf{k}} \phi\left(\mathbf{K}^{\prime}\right)\left[\cos \theta\left(\mathbf{K}^{\prime}\right)+1\right]\right] .
\end{aligned}
$$

Now, assuming that the attosecond pulse bandwidth is broad enough compared to the energy band and the initial excited population due to the attosecond x-ray pulse is similar over the whole conduction band, we can easily obtain Eq. (5).

[1] A. Schiffrin, T. Paasch-Colberg, N. Karpowicz, V. Apalkov, D. Gerster, S. Mühlbrandt, M. Korbman, J. Reichert, M. Schultze, S. Holzner, J. V. Barth, R. Kienberger, R. Ernstorfer, V. S. Yakovlev, M. I. Stockman, and F. Krausz, Optical-field-induced current in dielectrics, Nature (London) 493, 70 (2013).

[2] M. Schultze, E. M. Bothschafter, A. Sommer, S. Holzner, W. Schweinberger, M. Fiess, M. Hofstetter, R. Kienberger, V. Apalkov, V. S. Yakovlev, M. I. Stockman, and F. Krausz, Controlling dielectrics with the electric field of light, Nature (London) 493, 75 (2013).

[3] H. Mashiko, K. Oguri, T. Yamaguchi, A. Suda, and H. Gotoh, Petahertz optical drive with wide-bandgap semiconductor, Nat. Phys. 12, 741 (2016).

[4] M. Garg, M. Zhan, T. T. Luu, H. Lakhotia, T. Klostermann, A. Guggenmos, and E. Goulielmakis, Multi-petahertz electronic metrology, Nature (London) 538, 359 (2016).
[5] M. Baudisch, A. Marini, J. D. Cox, T. Zhu, F. Silva, S. Teichmann, M. Massicotte, F. Koppens, L. S. Levitov, F. J. García de Abajo, and J. Biegert, Ultrafast nonlinear optical response of Dirac fermions in graphene, Nat. Commun. 9, 1018 (2018).

[6] T. Higuchi, C. Heide, K. Ullmann, H. B. Weber, and P. Hommelhoff, Light-field-driven currents in graphene, Nature (London) 550, 224 (2017).

[7] J. Reimann, S. Schlauderer, C. P. Schmid, F. Langer, S. Baierl, K. A. Kokh, O. E. Tereshchenko, A. Kimura, C. Lange, J. Güdde, U. Höfer, and R. Huber, Subcycle observation of lightwave-driven Dirac currents in a topological surface band, Nature (London) 562, 396 (2018).

[8] C. Heide, T. Higuchi, H. B. Weber, and P. Hommelhoff, Coherent Electron Trajectory Control in Graphene, Phys. Rev. Lett. 121, 207401 (2018). 
[9] C. Heide, T. Boolakee, T. Higuchi, H. B. Weber, and P. Hommelhoff, Interaction of carrier envelope phase-stable laser pulses with graphene: the transition from the weak-field to the strong-field regime, New J. Phys. 21, 045003 (2019).

[10] H. Liu, Y. Li, Y. S. You, S. Ghimire, T. F. Heinz, and D. A. Reis, High-harmonic generation from an atomically thin semiconductor, Nat. Phys. 13, 262 (2017).

[11] T. T. Luu and H. J. Wörner, Measurement of the Berry curvature of solids using high-harmonic spectroscopy, Nat. Commun. 9, 916 (2018).

[12] R. E. F. Silva, I. V. Blinov, A. N. Rubtsov, O. Smirnova and M. Ivanov, High-harmonic spectroscopy of ultrafast many-body dynamics in strongly correlated systems, Nat. Photonics 12, 266 (2018).

[13] A. Chacón, D. Kim, W. Zhu, S. P. Kelly, A. Dauphin, E. Pisanty, A. S. Maxwell, A. Picón, M. F. Ciappina, D. E. Kim, C. Ticknor, A. Saxena, and M. Lewenstein, Circular dichroism in high-order harmonic generation: Heralding topological phases and transitions in Chern insulators, Phys. Rev. B 102, 134115 (2020).

[14] F. Langer, C. P. Schmid, S. Schlauderer, M. Gmitra, J. Fabian, P. Nagler, C. Schüller, T. Korn, P. G. Hawkins, J. T. Steiner, U. Huttner, S. W. Koch, M. Kira, and R. Huber, Lightwave valleytronics in a monolayer of tungsten diselenide, Nature (London) 557, 76 (2018).

[15] M. Hohenleutner, F. Langer, O. Schubert, M. Knorr, U. Huttner, S. W. Koch, M. Kira, and R. Huber, Real-time observation of interfering crystal electrons in high-harmonic generation, Nature (London) 523, 572 (2015).

[16] T. T. Luu, M. Garg, S. Yu. Kruchinin, A. Moulet, M. Th. Hassan, and E. Goulielmakis, Extreme ultraviolet highharmonic spectroscopy of solids, Nature (London) 521, 498 (2015).

[17] A. L. Cavalieri, N. Müller, T. Uphues, V. S. Yakovlev, A. Baltuska, B. Horvath, B. Schmidt, L. Blümel, R. Holzwarth, S. Hendel, M. Drescher, U. Kleineberg, P. M. Echenique, R. Kienberger, F. Krausz, and U. Heinzmann, Attosecond spectroscopy in condensed matter, Nature (London) 449, 1029 (2007).

[18] F. Siek, S. Neb, P. Bartz, M. Hensen, C. Strüber, S. Fiechter, M. Torrent-Sucarrat, V. M. Silkin, E. E. Krasovskii, N. M. Kabachnik, S. Fritzsche, R. Díez Muiño, P. M. Echenique, A. K. Kazansky, N. Müller, W. Pfeiffer, and U. Heinzmann, Angular momentum-induced delays in solid-state photoemission enhanced by intra-atomic interactions, Science 357, 1274 (2017).

[19] M. Ossiander, J. Riemensberger, S. Neppl, M. Mittermair, M. Schäffer, A. Duensing, M. S. Wagner, R. Heider, M. Wurzer, M. Gerl, M. Schnitzenbaumer, J. V. Barth, F. Libisch, C. Lemell, J. Burgdörfer, P. Feulner, and R. Kienberger, Absolute timing of the photoelectric effect, Nature (London) 561, 374 (2018).

[20] R. Locher, L. Castiglioni, M. Lucchini, M. Greif, L. Gallmann, J. Osterwalder, M. Hengsberger, and U. Keller, Energydependent photoemission delays from noble metal surfaces by attosecond interferometry, Optica 2, 405 (2015).

[21] Z. Tao, C. Chen, T. Szilvási, M. Keller, M. Mavrikakis, H. Kapteyn, and M. Murnane, Direct time-domain observation of attosecond final-state lifetimes in photoemission from solids, Science 353, 62 (2016).
[22] M. Schultze, K. Ramasesha, C. D. Pemmaraju, S. A. Sato, D. Whitmore, A. Gandman, J. S. Prell, L. J. Borja, D. Prendergast, K. Yabana, D. M. Neumark, and S. R. Leone, Attosecond bandgap dynamics in silicon, Science 346, 1348 (2014).

[23] S. R. Leone and D. M. Neumark, Attosecond science in atomic, molecular, and condensed matter physics, Faraday Discuss. 194, 15 (2016).

[24] M. Lucchini, S. A. Sato, A. Ludwig, J. Herrmann, M. Volkov, L. Kasmi, Y. Shinohara, K. Yabana, L. Gallmann, and U. Keller, Attosecond dynamical Franz-Keldysh effect in polycrystalline diamond, Science 353, 916 (2016).

[25] M. Zürch, H. T. Chang, L. J. Borja, P. M. Kraus, S. K. Cushing, A. Gandman, C. J. Kaplan, M. H. Oh, J. S. Prell, D. Prendergast, C. D. Pemmaraju, D. M. Neumark, and S. R. Leone, Direct and simultaneous observation of ultrafast electron and hole dynamics in germanium, Nat. Commun. 8, 15734 (2017).

[26] A. Moulet, J. B. Bertrand, T. Klostermann, A. Guggenmos, N. Karpowicz, and E. Goulielmakis, Soft x-ray excitonics, Science 357, 1134 (2017).

[27] F. Schlaepfer, M. Lucchini, S. A. Sato, M. Volkov, L. Kasmi, N. Hartmann, A. Rubio, L. Gallmann, and U. Keller, Attosecond optical-field-enhanced carrier injection into the GaAs conduction band, Nat. Phys. 14, 560 (2018).

[28] M. Volkov, S. A. Sato, F. Schlaepfer, L. Kasmi, N. Hartmann, M. Lucchini, L. Gallmann, A. Rubio, and U. Keller, Attosecond screening dynamics mediated by electron localization in transition metals, Nat. Phys. 15, 1145 (2019).

[29] B. Buades, A. Picon, E. Berger, I. Leon, N. Di Palo, S. L. Cousin, C. Cocchi, E. Pellegrin, J. H. Martin, S. Mañas-Valero, E. Coronado, T. Danz, C. Draxl, M. Uemoto, K. Yabana, M. Schultze, S. Wall, M. Zürch, and J. Biegert, Attosecond stateresolved carrier motion in quantum materials probed by soft X-ray XANES, arXiv:1808.06493.

[30] S. M. Teichmann, F. Silva, S. L. Cousin, M. Hemmer, and J. Biegert, 0.5-keV Soft x-ray attosecond continua, Nat. Commun. 7, 11493 (2016).

[31] D. Popmintchev, B. R. Galloway, M.-C. Chen, F. Dollar, C. A. Mancuso, A. Hankla, L. Miaja-Avila, G. O’Neil, J. M. Shaw, G. Fan, S. Alisauskas, G. Andriukaitis, T. Balciunas, O. D. Mücke, A. Pugzlys, A. Baltuska, H. C. Kapteyn, T. Popmintchev, and M. M. Murnane, Near- and Extended-Edge X-Ray-Absorption Fine-Structure Spectroscopy Using Ultrafast Coherent HighOrder Harmonic Supercontinua, Phys. Rev. Lett. 120, 093002 (2018).

[32] B. Buades, D. Moonshiram, T. P. H. Sidiropoulos, I. León, P. Schmidt, I. Pi, N. Di Palo, S. L. Cousin, A. Picón, F. Koppens, and J. Biegert, Dispersive soft X-ray absorption fine-structure spectroscopy in graphite with an attosecond pulse, Optica 5, 502 (2018).

[33] S. Huang, Y. Ding, Y. Feng, E. Hemsing, Z. Huang, J. Krzywinski, A. A. Lutman, A. Marinelli, T. J. Maxwell, and D. Zhu, Generating Single-Spike Hard X-Ray Pulses with Nonlinear Bunch Compression in Free-Electron Lasers, Phys. Rev. Lett. 119, 154801 (2017).

[34] S. Serkez, G. Geloni, S. Tomin, G. Feng, E. V. Gryzlova, A. N. Grum-Grzhimailo, and M. Meyer, Overview of options for generating high-brightness attosecond $\mathrm{x}$-ray pulses at freeelectron lasers and applications at the European XFEL, J. Opt. 20, 024005 (2018). 
[35] C. D. Pemmaraju, Simulation of attosecond transient soft x-ray absorption in solids using generalized Kohn-Sham real-time TDDFT, New J. Phys. 22, 083063 (2020).

[36] A. Picón, L. Plaja, and J. Biegert, Attosecond x-ray transient absorption in condensed-matter: A core-state-resolved Bloch model, New J. Phys. 21, 043029 (2019).

[37] O. Zurrón-Cifuentes, A. Picón, and L. Plaja, Theory of highorder harmonic generation for gapless graphene, New J. Phys. 20, 053033 (2018).

[38] O. Zurrón-Cifuentes, R. Boyero-García, C. Hernández-García, A. Picón, and L. Plaja, Optical anisotropy of non-perturbative high-order harmonic generation in gapless graphene, Opt. Express 27, 7776 (2019).

[39] M. Wu, S. Chen, S. Camp, K. J. Schafer, and M. B. Gaarde, Theory of strong-field attosecond transient absorption, J. Phys. B: At. Mol. Opt. Phys. 49, 062003 (2016).
[40] S. A. Sato, M. Lucchini, M. Volkov, F. Schlaepfer, L. Gallmann, U. Keller, and A. Rubio, Role of intraband transitions in photocarrier generation, Phys. Rev. B 98, 035202 (2018).

[41] C. Ott, A. Kaldun, P. Raith, K. Meyer, M. Laux, J. Evers, C. H. Keitel, C. H. Greene, and T. Pfeifer, Lorentz meets fano in spectral line shapes: A universal phase and its laser control, Science 340, 716 (2013).

[42] C. Ott, A. Kaldun, L. Argenti, P. Raith, K. Meyer, M. Laux, Y. Zhang, A. Blättermann, S. Hagstotz, T. Ding, R. Heck, J. Madroñero, F. Martín, and T. Pfeifer, Reconstruction and control of a time-dependent two-electron wave packet, Nature (London) 516, 374 (2014).

[43] Y. Cheng, M. Chini, X. Wang, A. González-Castrillo, A. Palacios, L. Argenti, F. Martín, and Z. Chang, Reconstruction of an excited-state molecular wave packet with attosecond transient absorption spectroscopy, Phys. Rev. A 94, 023403 (2016). 